\title{
A REMARK ON THE EXPECTATIONS OF OPERATOR ALGEBRAS
}

\author{
By M. Nakamura, M. Takesaki and H. Umegaki
}

If a transformation $a \rightarrow a^{\varepsilon}$ defined on a $C^{*}$-algebra having the identity, which mapps it into itself, (satisfies; i) linear, ii) indempotent: $a^{\varepsilon \varepsilon}=a^{\varepsilon}$, iii) positive: $a^{\varepsilon} \geqq 0$ for $a \geqq 0$, iv) unit-preserving: $1^{\varepsilon}=1$, and moreover $\left(x^{\varepsilon} y\right)^{\varepsilon}=x^{\varepsilon} y^{\varepsilon}$, then $\varepsilon$ is said to be an expectation in the sense of [7]. Clearly, the notion of the expectation is an extension of that of traces, states, Dixmier's centering $\zeta$ etc. The endomorphism of Reynolds [5], which is defined on a functional algebra, is also an expectation. A detailed treatise on expectations of discrete finite factors will be found in a recent publication of Davis [3].

Among expectation, in a connection with the theory of probability, the conditional expectation in the sense of [11] has the special importance. In a von Neumann algebra $A$ with a faithful normal trace $\tau$, for a von Neumann subalgebra $B$ of $A$, the conditional expectation $\varepsilon$ conditioned by $B$ is defined as an operation which maps $a \in A$ to $a^{\varepsilon} \in B$ such that $\tau(a b)=\tau\left(a^{\varepsilon} b\right)$ for any $b \in B$. It is known that the conditional expectation in the sense of the above coincides with the usual one if the algebra $A$ is commutative (e.g., cf. [7] or [11]). It is also obvious that the conditional expectation is a projection which maps $A$ onto $B$ considering $A$ as a pre-Hilbert space introducing an inner product by the trace as usually.

The present note contains a proof that the conditional expectation in a not necessarily commutative probability is completely positive in the sense of Stinespring [8] or positive definite in the sense of [10]. Consequently two representation theorems on the conditional expectation follow. They will be proved in $\S 1$, and their generalizations on $C^{*}$-algebras will be discussed in $\S 3$ briefly.

The remainder of the note, $\$ 2$, contains a discussion to generalize Jensen's inequality for non-commutative probability. A formal extension of the operator convexity of Bendat-Sharman [1] allows us a formulation for finite continuous factors, by the help of Davis' theorem [2]. However, the final form still remains for.

1. Complete positivity of the conditional expectation.

A linear transformation $\theta$ defined on a $C^{*}$-algebra $A$ into an operator algebra

Received April 14, 1960. 
$B$ is called positive if $\theta$ satisfies

$$
x^{\theta} \geqq 0, \quad \text { for any } x \geqq 0,
$$

and $\theta$ is called completely positive in the sense of Stinespring [8], equivalently positive definite in the sense of $[10]$, if $\theta(n)$ :

$$
\left(x_{\jmath j}\right)^{\theta(n)}=\left(x_{\imath j}{ }^{\theta}\right),
$$

is positive on $n \times n$ matrix algebra over $A$, for every $n$.

Theorem 1. If $A$ is a von Neumann algebra of finite type with a faithful normal trace $\pi$ in the sense of Dixmier [4], and if $B$ is a von Neumann subalgebra of $A$, then the conditional expectation conditioned by $B$ is completely positive.

Proof. Consider the matrix algebra $C=I_{n} \otimes A$ for finite $n$ and its subalgebra $D=I_{n} \otimes B$ (cf. Dixmier [4]). In [4], its trace $\tau$ is given as usually by

$$
\tau\left[\left(a_{\imath \jmath}\right)\right]=\frac{1}{n} \sum_{i=1}^{n} \pi\left(a_{i i}\right)
$$

whence $C$ becomes a von Neumann algebra of finite type with a faithful normal trace $\tau$. Consequently, the conditional expectation $\div$ conditioned by $D$ will be introduced by

$$
\tau\left[\left(a_{\imath j}\right)\left(b_{i j}\right)\right]=\tau\left[\left(a_{\imath j}\right)-\left(b_{i j}\right)\right],
$$

for $\left(a_{\imath j}\right) \in C$ and $\left(b_{i j}\right) \in D$. By (3),

$$
\begin{aligned}
\tau\left[\left(a_{i j}\right)\left(b_{i j}\right)\right] & \left.=\tau\left[\sum_{k=1}^{n} a_{i k} b_{k j}\right)\right]=\frac{1}{n} \sum_{i, k} \pi\left(a_{i k} b_{k i}\right) \\
& =\frac{1}{n} \sum_{i, k} \pi\left(a_{i k}^{\varepsilon} b_{k i}\right)=\tau\left[\left(a_{i j}^{\varepsilon}\right)\left(b_{i j}\right)\right],
\end{aligned}
$$

and this implies $\tau\left[\left(a_{i j}\right)-\left(b_{i j}\right)\right]=\tau\left[\left(a_{i j}^{\varepsilon}\right)\left(b_{i j}\right)\right]$ for every $\left(a_{\imath \jmath}\right) \in C$ and $\left(b_{i j}\right) \in D$, whence

$$
\left(a_{i j}\right)^{-}=\left(a_{i j}^{\varepsilon}\right)
$$

for all $\left(a_{i j}\right) \in C$. Using the positivity of $\div$ in $C,\left(a_{i j}\right) \geqq 0$ implies $\left(a_{i j}\right)-\geqq 0$, whence $\left(a_{i j}^{\varepsilon}\right) \geqq 0$ by (5), which shows the complete positivity of the conditional expectation.

REMARK. Although Theorem 1 is proved for the conditional expectation, it remains true for a $B$-expectation: If the tracelet space $S_{B}$ in the sense of [11] is non-void, then a $B$-expectation $\varepsilon_{\sigma}$ defined by a tracelet $\sigma$ is completely positive as a mapping which carries $A$ onto $b_{\sigma} B$ where $b_{\sigma}$ is the $B$-support of $\sigma$.

Since the conditional expectation is completely positive by Theorem 1, Stinespring's theorem [8] implies at once a representation of the conditional expectation in Neumark's form: Let $K$ be the direct product $H \otimes_{\varepsilon} A$ in the 
sence of [10] which is the completion of the pre-Hilbert space of quotient space of the algebraic tensor product $H \odot A$ modulo the null space with respect to the inner product:

$$
\left\langle\sum_{\xi \in H, \alpha \in A} \xi \otimes a, \sum_{\xi^{\prime} \in \sum_{H, \alpha^{\prime} \in A}} \xi^{\prime} \otimes a^{\prime}\right\rangle=\sum_{\xi, \xi^{\prime} \in H, \alpha, \alpha^{\prime} \in A}\left(\xi,\left(a^{*} a^{\prime}\right)^{\varepsilon} \xi^{\prime}\right),
$$

where $(, \quad)$ is the inner product of $H$, and $\sum$ is the finite sum. Then $H \otimes_{\odot} B$ is a subspace of $H \otimes_{\varepsilon} A$ and it is equivalent to $H$ by the unitary transformation

$$
\sum_{\xi \in H, b \in B} \xi \otimes b \rightarrow \sum_{\xi \in H, b \in B} b \xi .
$$

Identifying $H \otimes_{\varepsilon} B$ with $H, H$ can be regarded as a subspace of $H \otimes_{\varepsilon} A$. Then Stinespring's theorem implies the following:

THEOREM 2. The conditional expectation $\varepsilon$ of a von Neumann algebra $A$ of finite type with a faithful normal trace is representable by

$$
a^{\varepsilon}=\widetilde{p} a^{\#} \tilde{p}, \quad \text { for every } a \in A,
$$

where $\tilde{p}$ is the projection of $H \otimes_{\varepsilon} A$ onto $H$ which belongs to the commutant

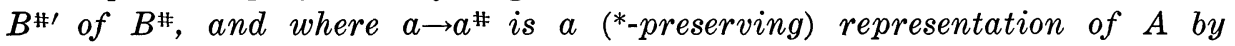
operators on $H \otimes_{\varepsilon} A$ defined by

$$
a^{\#}\left(\sum \xi \otimes a^{\prime}\right)=\sum \xi \otimes a a^{\prime} .
$$

The representation of $A$ is continuous ultra-weakly.

Proof. The equation (7) follows from Stinespring's theorem and Theorem 1. Putting $E\left(\sum \xi \otimes a\right)=\sum \xi \otimes a^{\varepsilon}$,

$$
\begin{aligned}
\left\langle E\left(\sum \xi \otimes a\right),\right. & \left.\sum \xi^{\prime} \otimes a^{\prime}\right\rangle=\left\langle\sum \xi \otimes a^{\varepsilon}, \sum \xi^{\prime} \otimes a^{\prime}\right\rangle \\
& =\sum\left(\xi,\left(a^{* \varepsilon} a^{\prime}\right)^{\varepsilon} \xi^{\prime}\right)=\sum\left(\xi,\left(a^{*} a^{\prime \varepsilon}\right)^{\varepsilon} \xi^{\prime}\right) \\
& =\left\langle\Sigma \xi \otimes a, \quad \sum \xi^{\prime} \otimes a^{\prime \varepsilon}\right\rangle=\left\langle\sum \xi \otimes a, E\left(\sum \xi^{\prime} \otimes a^{\prime}\right)\right\rangle,
\end{aligned}
$$

and

$$
E(E(\Sigma \xi \otimes a))=E\left(\Sigma \xi \otimes a^{\varepsilon}\right)=\Sigma \xi \otimes a^{\varepsilon}=E\left(\sum \xi \otimes a\right) .
$$

Furthermore,

$\left\langle E\left(\sum \xi_{i} \otimes a_{i}\right), E\left(\sum \xi_{i} \otimes a_{i}\right)\right\rangle=\left\langle\sum \xi_{i} \otimes a_{\imath}^{\varepsilon}, \quad \sum \xi_{i} \otimes a_{\imath}{ }^{\varepsilon}\right\rangle$

$$
\begin{aligned}
& =\sum_{\imath, j}\left(\xi_{i}, a_{\imath}{ }^{* \varepsilon} a_{j}^{\varepsilon} \xi_{j}\right)=\left\langle\left(\begin{array}{c}
\xi_{1} \\
\vdots \\
\xi_{n}
\end{array}\right),\left(a_{\imath}{ }^{* \varepsilon} a_{j}^{\varepsilon}\right)\left(\begin{array}{c}
\xi_{1} \\
\vdots \\
\xi_{n}
\end{array}\right)\right\rangle
\end{aligned}
$$

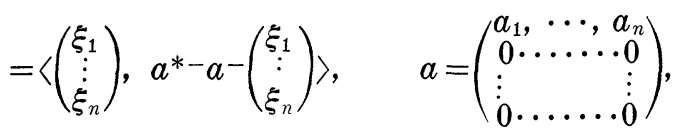




$$
\begin{aligned}
& \leqq\left\langle\left(\begin{array}{c}
\xi_{1} \\
\vdots \\
\xi_{n}
\end{array}\right),\left(a^{*} a\right)-\left(\begin{array}{c}
\xi_{1} \\
\vdots \\
\xi_{n}
\end{array}\right)\right\rangle=\sum_{\imath, j}\left(\xi_{i},\left(a_{\imath}{ }^{*} a_{j}\right)^{\varepsilon} \xi_{j}\right) \\
& =\left\langle\Sigma \xi_{i} \otimes a_{\imath}, \sum \xi_{i} \otimes a_{\imath}\right\rangle .
\end{aligned}
$$

Therefore $E$ is well defined and is uniquely extended to the projection $\tilde{p}$ on $H \otimes_{\varepsilon} A$, and

$$
\begin{aligned}
b^{\#} \tilde{p} \sum \xi \otimes a & =b^{\#} \sum \xi \otimes a^{\varepsilon}=\Sigma \xi \otimes b a^{\varepsilon}=\Sigma \xi \otimes(b a)^{\varepsilon} \\
& =\tilde{p} \Sigma \xi \otimes b a=\tilde{p} b^{\#} \sum \xi \otimes a,
\end{aligned}
$$

and we obtain $\tilde{p} \in B^{\# \prime}$. The ultra-weak continuity is obvious from the definition of the representation $a \rightarrow a^{\#}$.

If the algebra $A$ is commutative, i. e., $(A, \pi)$ is a pair of all bounded random variables and the expectation (for instance, cf. [11]), then $A$ (resp. $B$ ) is isometrically isomorphic with the algebra $C(\Omega)$ (resp. $C(\Gamma)$ ) of all continuous functions defined on the compact spectrum $\Omega$ of $A$ (resp. $\Gamma$ of $B$ ). Denote the isomorphism by $a \rightarrow a(\omega)$ (resp. $b \rightarrow(\gamma)$ ) between $A$ and $C(\Omega)$ (resp. $B$ and $C(\Gamma)$ ), further denote $\omega^{\prime}=\omega \mid B$, the restriction of $\omega$ onto $B$ as a linear function of $B$, then it is a pure state of $B$ and is considerable as a point of $\Gamma$. The mapping $\omega \rightarrow \omega^{\prime}$ is continuous from $\Omega$ onto $\Gamma$. Under these notations, we have followings. Firstly, Theorem 2 implies the following

THEOREM 3. The conditional expectation $\varepsilon$ of a commutative von Neumann algebra conditioned by a certain von Neumann subalgebra $B$ is representable by an integral formulae:

$$
a^{\varepsilon}=\int_{\Omega} a(\omega) d F(\omega) \quad \text { for every } a \in A,
$$

where $d F$ is an operator measure of Neumark which is defined over the Borel field of $\Omega$ and valued in $B$.

Proof. The *-representation $a \rightarrow a^{\#}$ of $A$ is represented by a spectral measure $d E$ on $H \otimes_{\varepsilon} A$ :

$$
a^{\#}=\int_{\Omega} a(\omega) d E_{\omega} \quad \text { for every } a \in A
$$

Therefore

$$
\begin{aligned}
\left\langle a^{\xi} \sum \xi \otimes b,\right. & \left.\sum \xi^{\prime} \otimes b^{\prime}\right\rangle=\left\langle a^{\#} \sum \xi \otimes b, \sum \xi^{\prime} \otimes b^{\prime}\right\rangle \\
& =\int_{\Omega} a(\omega) d\left\langle E_{\omega} \Sigma \xi \otimes b, \quad \sum \xi^{\prime} \otimes b^{\prime}\right\rangle \\
& =\int_{\Omega} a(\omega) d\left\langle\widetilde{p} E_{\omega} \tilde{p} \Sigma \xi \otimes b, \quad \sum \xi^{\prime} \otimes b^{\prime}\right\rangle .
\end{aligned}
$$


Putting $\widetilde{p} E \widetilde{p}=F,(10)$ implies (9). The operators $F_{S}$, for every Borel set $S$ in $\Omega$, clearly belongs to $B$.

This theorem is a generalization of a theorem of [6]: If $h$ is a linear functional of $A$ invariant under $\varepsilon$ in the sense of $h\left(a^{s}\right)=h(a)$, then there exists a regular Borel measure $\nu$ on $\Omega$ such that

$$
h(a)=\int_{r^{\prime}}(a, \gamma) d \nu(\gamma) \quad \text { for every } a \in A,
$$

where $(a, \gamma)=a^{s}(\gamma)$. Indeed, this follows from Theorem 3, or more precisely from the following formulae obtained from the formulae (9):

$$
a^{\varepsilon}=a^{\varepsilon \varepsilon}=\int_{\Omega} a^{\varepsilon}(\omega) d F(\omega)=\int_{I^{\prime}}(a, \gamma) d F(\gamma)
$$

where $d F(\gamma)$ is the operator measure over the Borel field of $\Gamma$ induced by $d F(\omega)$.

According to the usual language of probability theory, Theorem 3 can be read as following:

THEOREM 3'. If $(\Omega, \mathfrak{A})$ is a probability field with probability measure pr. and if $\mathfrak{B}$ is a Borel subfield of $\mathfrak{A}$, then the conditional probability $P(S \mid \mathfrak{B})$ is expressible as

$$
P(S \mid \mathfrak{B})(.)=\left(\int_{S} d F(\omega)\right)(.) \quad \text { for every } S \in \mathfrak{A},
$$

where $d F$ is an operator measure of Neumark whose values are operators on $L^{2}(\Omega, \mathfrak{A}, p r$.$) .$

2. Jensen's inequality in non-commutative probability.

If $f(\lambda)$ is a continuous real-valued function defined on an interval $[\alpha, \beta]$, and if $a$ is an hermitean operator:

$$
a=\int_{\alpha}^{\beta} \lambda d e_{\lambda}
$$

then there is an hermitean operator

$$
f(a)=\int_{\alpha}^{\beta} f(\lambda) d e_{\lambda} .
$$

Therefore, if $A$ is a $C^{*}$-algebra (assuming to have the identity), then the mapping $a \rightarrow f(a)$, defined by (12), carries hermitean elements with the spectra in $[\alpha, \beta]$ into hermitean elements of the algebra. This mapping preserves some properties of $f$ when $A$ is commutative: For example, if $f$ is monotone then

$$
a \leqq b \quad \text { implies } f(a) \leqq f(b),
$$


and if $f$ is convex then

$$
f(\lambda a+(1-\lambda) b) \leqq \lambda f(a)+(1-\lambda) f(b), \quad 0 \leqq \lambda \leqq 1,
$$

for every hermitean elements $a$ and $b$ having their spectra in the interval. Whereas, if the algebra $A$ is not commutative, the situation changes. Even if the algebra $A$ is the total matrix algebra of finite order $n$, a monotone [convex] $f$ does not sftisfy (13) $[(14)]$ in general. The class of all (continuous real-valued) function $f$ satisfying (13) $[(14)]$ is known as Löwner's monotone [convex] matrix functions of order $n$, which is strictly restrictive than the monotonity [convexity]. When $A$ is the algebra of all operators on an infinite dimensional Hilbert space, the class of all functions satisfying (13) [(14)] are studied recently by Bendat-Sherman [1]. They showed, among many others, a continuous real-valued function satisfies (13) $[(14)]$ if and only if it is matrix monotone [convex] in arbitrary order.

It is troublesome that the operator convexity or monotonity do not coincide with the usual. For example, there is no way to generalize Jensen's inquality including both commutative and non-commutative cases. To avoid the difficulty, the note proposed a trial introducing the following

Definition. A real-valued continuous function $f(\lambda)$ defined on an interval is a monotone [convex] operator function with respect to a $C^{*}$-algebra $A$, abbreviatively A-monotone [A-convex], provided that (13) [(14)] holds for any hermitean members $a$ and $b$ of $A$ having their spectra in the interval.

It is obvious that the following properties are true:

(i) a constant function is always $A$-convex,

(ii) $f+g$ is $A$-convex when $f$ and $g$ are $A$-convex,

(iii) a convex function is operator convex with respect to a commutative algebra $A$,

(iv) an operator convex function in the sense of Löwner-Bendat-Sherman is operator convex with respect to the algebra of all operators on an infinite dimensional Hilbert space.

Consequently, (iii) and (iv) imply that the definition covers both cases. However, it seems hopeless, that the definition yields substantially a new class of functions, which may be shown by the following example:

(v) If $f$ is operator convex with respect to a continuous finite factor $A$, then $f$ is operator convex in the sense of Löwner-Bendat-Sherman.

This is because of that a continuous finite factor always contains a subfactor which is isomorphic to a discrete finite factor of arbitrary order.

Except these rather trivial cases (iii)-(iv), it is unable to decide in the present knowledge that $A$-convexity coincides with the convexity of LöwnerBendat-Sherman or not. This affects to the incompleteness of the following theorem which seems to the authors a non-commutative extension of Jensen's inequality: 
THEOREM 4. If $A$ is von Neumann algebra of finite type with a faithful normal trace which is either commutative or a continuous factor, if $f$ is operator convex with respect to $A$ on an interval, and if $\varepsilon$ is the conditional expectation conditioned by a certain von Neumann subalgebra of $A$, then the following "Jensen's inequality" holds:

$$
f\left(a^{\varepsilon}\right) \leqq f(a)^{\varepsilon},
$$

for any hermitean member a of $A$ which has its spectrum in the interval.

Proof. If $A$ is commutative then the theorem is Jensen's inequality itself. If $A$ is a finite and continuous factor, then it is not less general to assume that $f$ satisfies by

$$
f(0)=0,
$$

by (i)-(ii), whence the theorem follows from (v), Theorem 1 and a theorem due to Davis [2].

REMARK. If $\varepsilon$ is an expectation of a $C^{*}$-algebra $A$ (having the identity) in the sense of [7], and if $f$ is a general mapping of $A$ into itself which preserves also $A^{\varepsilon}$, then (15) holds when $\varepsilon$ and $f$ satisfy

$$
f\left(a^{\varepsilon}\right) \leqq f(a),
$$

since $f\left(a^{\varepsilon}\right)^{\varepsilon}=f\left(a^{\varepsilon}\right)$ by the idempotency of the expectation and since $f\left(a^{\varepsilon}\right)^{\varepsilon} \leqq f(a)^{\varepsilon}$ by the positiveness of the expectation. The case really occurs, as recently proved by Davis [3], when $\varepsilon$ is a "pinching" of a discrete finite factor and $f$ is Schur-convex.

\section{Complete positivity of expectations in a $C^{*}$-algebra.}

Theorems 1-3 are almost valid when an expectation in the sense of [7] is employed in place of the conditional expectation. Major modification occurs in Theorem 1 since the presence of the trace is not assumed, whence Theorem 1 in case of $C^{*}$-algebras needs to restate and to generalize in the following

Theorem 5. If $A$ is a $C^{*}$-algebra with the identity and if $\varepsilon$ is an expectation of $A$, then $\varepsilon$ is completely positive.

Proof. Put $C=I_{n} \otimes A$ and $D=I_{n} \otimes B$ again, where $B$ is the range of $\varepsilon$. They are $C^{*}$-algebras too, and $C$ contains $D$ as a subalgebra. $A$ mapping $\div$ defined by

$$
\left(\sum x_{i} \otimes a_{i}\right)^{-}=\sum x_{i} \otimes a_{i}^{\varepsilon}, \quad x_{i} \in I_{n}, a_{i} \in A,
$$

is a projection from $C$ onto $D$. Put $h^{\varepsilon}(a)=h\left(a^{\varepsilon}\right)$ for $h \in A^{*}$ and $\left(A^{*}\right)^{\varepsilon}=\left\{h^{\varepsilon} \mid h \in A^{*}\right\}$, where $E^{*}$ means the conjuagate of a Banach space $E$. Then 


$$
\begin{aligned}
& \left\|h^{\varepsilon}\right\|_{A} \text { (the norm as functional of } A \text { ) } \\
= & \sup _{0 \neq a, a \in A}\left|h^{\varepsilon}(a)\right| /\|a\| \leqq \sup _{\substack{0 \neq \alpha, \alpha \in A \\
=}}\left|h^{\varepsilon}\left(a^{\varepsilon}\right)\right| /\left\|a^{\varepsilon}\right\| \\
= & \left\|h^{\varepsilon}\right\|_{B} \leqq\left\|h^{\varepsilon}\right\|_{A} .
\end{aligned}
$$

Since the correspondence $g \in\left(A^{*}\right)^{\varepsilon} \rightarrow g \mid B \in B^{*}$, the restriction of $g$ onto $B$ maps $\left(A^{*}\right)^{\varepsilon}$ onto $B^{*}$, it is an isometric isomorphism between $\left(A^{*}\right)^{\varepsilon}$ and $B^{*}$. Therefore the norm of the mapping $\div$ is one:

$$
\begin{aligned}
& \left\|\left(\sum x_{i} \otimes a_{i}\right)^{-}\right\|=\left\|\sum x_{i} \otimes a_{i}^{\varepsilon}\right\| \\
= & \sup _{y_{j} \in I_{n^{*}, g_{j} \in B^{*}}}\left|\left(\sum y_{j} \otimes g_{j}\right)\left(\sum x_{i} \otimes a_{i}^{\varepsilon}\right)\right| /\left\|\sum y_{i} \otimes g_{j}\right\| \\
= & \sup _{y_{j} \in I n^{*}, g_{j} \in\left(A^{*}\right) \varepsilon}\left|\left(\sum y_{j} \otimes g_{j}\right)\left(\sum x_{i} \otimes a_{i}^{\varepsilon}\right)\right| /\left\|\sum y_{j} \otimes g_{j}\right\| \\
= & \sup _{y_{j} \in I_{n^{*}, g_{j} \in\left(A^{*}\right) \varepsilon}}\left|\left(\sum y_{j} \otimes g_{j}\right)\left(\sum x_{i} \otimes a_{i}\right)\right| /\left\|\sum y_{j} \otimes g_{j}\right\| \\
\leqq & \sup _{y_{j} \in I_{n^{*}, h_{j} \in A^{*}}}\left|\left(\sum y_{i} \otimes h_{j}\right)\left(\sum x_{i} \otimes a_{i}\right)\right| /\left\|\sum y_{j} \otimes h_{j}\right\|,
\end{aligned}
$$

because $\left(A^{*}\right)^{\varepsilon}$ is a subspace of $A^{*}$,

$$
=\left\|\sum x_{i} \otimes a_{i}\right\| \text {. }
$$

Therefore $\div$ is positive by a theorem of Tomiyama [9; Them. 1$]$, which proves the theorem.

Theorem 2 and 3 (except the ultra-weak continuity of the representation) are still true for an expectation of a $C^{*}$-algebra. Their proofs need few modifications, whence they will be omitted here.

\section{REFERENCES}

[1] Bendat, J., AND Shereman, Mnonotone and convex operator functions. Trans. Amer. Math. Soc. 79 (1951), 58-71.

[2] Davis, Chandler, A Schwarz inequality for convex operator functions. Proc. Amer. Math. Soc. 8 (1957), 42-44.

[3] Davis, Chander, Various averaging operations onto subalgebras, Ill. J. Math. 3 (1959), 538-553.

[4] Dixmier, J., Les algèbres d'opérateurs dans l'espace hilbertien. Paris, 1957.

[5] Dubreil-JAcotin, J.-L., Etude algébrique des transformations de Reynolds. Colloque d'Algèbre Bruxelles, December, 1956.

[6] Nakamura, M., A remark on the integral decomposition of a measure. Mem. Osaka Univ. Liberal-Arts and Ed. B 3 (1954), 25-28.

[7] Nakamura, M., AND T. TURUMARU, Expectations in an operator algebra. Tôhoku Math. J. 6 (1954), 182-188.

[8] Stinespring, W.F., Positive functions on $C^{*}$-algebras. Proc. Amer. Math. Soc. 6 (1955), 211-216.

[9] Tомiчama, J., On the projection of norm one in $W^{*}$-algebras. Proc. Jap. Acad. 33 (1957), 608-612. 
[10] Umegaki, H., Positive definite functions and direct product Hilbert space. Tôhoku Math. J. 7 (1955), 206-211.

[11] UmegakI, H., Conditional expectation in an operator algebra, I-III. Tôhoku Math. J. 6 (1954), 177-181; 8 (1956), 86-100; Kōdai Math. Sem. Rep. 11 (1959), 51-64.

OSAKa University of Libral Arts and Education, ToKyo Institute of TeChNOLOGY, AND

Tokyo Institute of Technology. 